\title{
Mindfulness and pharmacological prophylaxis after withdrawal from medication overuse in patients with Chronic Migraine: an effectiveness trial with a one-year follow-up
}

Licia Grazzi $i^{*}$, Emanuela Sansone ${ }^{2}$, Alberto Raggi ${ }^{2}$, Domenico D'Amico ${ }^{1}$, Andrea De Giorgio ${ }^{3}$, Matilde Leonardi², Laura De Torres ${ }^{2}$, Francisco Salgado-García ${ }^{4}$ and Frank Andrasik ${ }^{4}$

\begin{abstract}
Background: Chronic Migraine (CM) is a disabling condition, worsened when associated with Medication Overuse (MO). Mindfulness is an emerging technique, effective in different pain conditions, but it has yet to be explored for CM-MO. We report the results of a study assessing a one-year course of patients' status, with the hypothesis that the effectiveness of a mindfulness-based approach would be similar to that of conventional prophylactic treatments.

Methods: Patients with CM-MO (code 1.3 and 8.2 of the International Classification of Headache Disorders-3Beta) completed a withdrawal program in a day hospital setting. After withdrawal, patients were either treated with Prophylactic Medications (Med-Group), or participated in a Mindfulness-based Training (MT-Group). MT consisted of 6 weekly sessions of guided mindfulness, with patients invited to practice 7-10 min per day. Headache diaries, the headache impact test (HIT-6), the migraine disability assessment (MIDAS), state and trait anxiety (STAI Y1-Y2), and the Beck Depression Inventory (BDI) were administered before withdrawal and at each follow-up $(3,6,12$ after withdrawal) to patients from both groups. Outcome variables were analyzed in separate two-way mixed ANOVAs (Group: Mindfulness vs. Pharmacology x Time: Baseline, 3-, 6-, vs. 12-month follow-up).
\end{abstract}

Results: A total of 44 patients participated in the study, with the average age being 44.5 , average headache frequency/ month was 20.5, and average monthly medication intake was 18.4 pills. Data revealed a similar improvement over time in both groups for Headache Frequency (approximately 6-8 days reduction), use of Medication (approximately 7 intakes reduction), MIDAS, HIT-6 (but only for the MED-Group), and BDI; no changes on state and trait anxiety were found. Both groups revealed significant and equivalent improvement with respect to what has become a classical endpoint in this area of research, i.e. $50 \%$ or more reduction of headaches compared to baseline, and the majority of patients in each condition no longer satisfied current criteria for CM.

Conclusions: Taken as a whole, our results suggest that the longitudinal course of patients in the MT-Group, that were not prescribed medical prophylaxis, was substantially similar to that of patients who were administered medical prophylaxis.

Keywords: Chronic migraine, Medication overuse, Pharmacological prophylaxis, Mindfulness

\footnotetext{
* Correspondence: licia.grazzi@istituto-besta.it

"Neurological Institute "C. Besta" IRCCS Foundation, Headache and

Neuroalgology Unit, Via Celoria 11, 20133 Milan, Italy

Full list of author information is available at the end of the article
} 


\section{Background}

Headache disorders are common disabling conditions that, in the last Global Burden of Disease study, were rated as the sixth cause of disability [1]. Further, medication overuse headache was rated as the $18^{\text {th }}$ and, among those aged under 50, migraine was rated as the third cause of disability [2]. Chronic Migraine (CM), is construed as a negative evolution of episodic migraine, based on the findings that approximately $2.5 \%$ of episodic migraineurs progress to CM each year [3], with a prevalence of approximately $2 \%[3,4]$. CM is diagnosed when headache episodes occur more than 15 days/ month (with at least 8 displaying migraine headache features) for more than three months [5], and is frequently associated with overuse of acute medications. This has been hypothesized to be one of the chief factors contributing to migraine chronification $[6,7]$ and, when overuse of medicine reaches a level to warrant a diagnosis of Medication Overuse (MO), it further complicates CM making it particularly difficult to manage. Chronic Migraine associated with Medication Overuse (CM-MO) is diagnosed when the intake of headache medications for headache episodes is greater than 15 days/month for simple analgesics, or exceeds 10 days/month for triptans, opioids, ergotamine or combinations of certain drugs [5]. A recent review of the literature evidenced that analgesics and opioids are associated with a higher risk of developing MO and the authors concluded that the socalled "migraine-specific" treatments, namely triptans and ergots, should be preferred as they are less frequently associated with development of overuse and disease chronification [8].

Patients with CM-MO present therapeutic challenges and require multidisciplinary care, including pharmacological and non-pharmacological therapeutic approaches [9]. Various pharmacological therapies have more recently been developed to help these patients better manage their condition [10-13], but symptom resolution is not always optimal and up to one third of patients relapse by 12 months [14-16]. Medication withdrawal is strongly recommended and its use can be viewed as a "reset" that then affords patients a greater likelihood of positively responding to appropriate prophylactics [13, 17, 18]. It is most helpful when patients are provided education and support about proper use of medications and taught strategies for avoiding relapse [19]. Studies have shown that such approaches can produce significant improvements that endure for extended periods, up to 5 years $[20,21]$.

$\mathrm{CM}-\mathrm{MO}$ results in pervasive negative consequences, where personal suffering is accompanied by reduced quality of life and disability, and decreased abilities to participate in daily work and/or family activities, which often results in increasing symptoms of depression [12, 22-24]. Clinicians and researchers working in the field of headache disorders are becoming increasingly aware of the consequences of this condition, the resultant need for a multifactorial approach and treatment [25]. The joint use of pharmacological and non-pharmacological techniques has been shown to improve the health status of migraine patients and to enhance clinical outcomes by teaching and reinforcing patients to implement alternative procedures for addressing and coping with headache attacks [9, 26-30].

Among the wide array of available non-pharmacological treatments, mindfulness has been recently included in rehabilitation programs for chronic pain conditions [31-36]. Its efficacy has been addressed in a recent review of the psychological therapies in the neurorehabilitation of pain syndromes [37], where it has been judged as effective (Grade Level A) for chronic pain syndromes with heterogeneous physiopathology, exclusive of headache disorders. However when this review was prepared researchers had only begun to explore the utility of mindfulness for headache, so no firm recommendations could be made. The success with other pain conditions, however, has spurred researchers in the field of headache to increasingly turn their attention to mindfulness training as another viable alternative approach for supplementing patient care. The main goal of this approach is to increase patient awareness of their pain and improve their abilities to manage headache before resorting to their former medications [38-45]. As concluded in two recent reviews on the use of mindfulness-based approaches in headache disorders $[46,47]$, this kind of approach seems promising. In brief, literature findings [40-45] suggest that various mindfulness-based approaches may be helpful for headache sufferers, and that it may be of value also for those with CM-MO. However, the available studies are limited by an inadequate consideration of some of the most important endpoints in chronic headache research, namely the frequency of headache and the consumption of medications for acute headaches management. Further, other meaningful indicators of effectiveness, such as pain intensity, headache duration, disability, quality of life and some mental health-related variables, such as stress, anxiety, pain acceptance or self-efficacy, have yet to be fully explored. A second relevant shortcoming is the limited duration of follow-up reported in these studies, which has ranged between 3 weeks and 3 months. Finally, what is not clear is the ability of a mindfulness-based approach by itself to impact key primary as well as secondary migraine headache parameters, as well as promote reductions in consumption of acute medications. Two research areas, thus, warrant further attention: first, identifying optimal components and 
delivery schedules, by adequately specifying the intervention protocols; second, conducting rigorous controlled trials that assess the durability of effects over extended time periods, with appropriate control conditions and a clear specification of primary and secondary outcomes.

As a way to begin to address these uncertainties we conducted an exploratory clinical trial, one that compared conventional prophylactic pharmacological treatment alone to a mindfulness-based treatment alone for patients diagnosed with $\mathrm{CM}-\mathrm{MO}$ and incorporated a more extended follow-up period. We carefully monitored the clinical course of these patients after all had undergone a structured withdrawal, with the hypothesis that the mindfulness-based approach would be similar in effectiveness when compared to conventional prophylactic treatment, for reducing headache frequency, consumption of acute medications, headache impact, symptoms of depression, and of anxiety.

\section{Methods \\ Participants}

Eligible patients were those diagnosed with CM-MO i.e. code 1.3 and associated medication overuse, following the international criteria included in point 8.2 of the International Classification of Headache Disorder III edition, beta version (ICHD-3-beta) [5] - who presented consecutively for treatment at the Headache Centre of the Neurological Institute C. Besta of Milan, Italy, between February 2014 and June 2015. These patients were aged between 18 and 65 years and had a history of CM lasting for at least ten years that was associated with overuse of Triptans and non-steroidal anti-inflammatory drugs (NSAIDs) for a minimum of the past five years. Patients with comorbid major psychiatric disorders, namely psychotic disorders and personality disorders, determined on the basis of clinical history and psychiatric evaluation, or pregnancy were excluded.

\section{Procedures}

All patients were first admitted to our out-patient day hospital service, where they participated in a 5-day structured medication withdrawal program that utilized intravenous therapy, including steroids and ademetionine [28, 48]. During withdrawal, patients were instructed to avoid the use of medications to manage any acute pain attacks. Upon completion of the structured medication withdrawal, all patients were encouraged to increase their physical activity and perform aerobic exercises, for $45 \mathrm{~min}$ twice per week, maintain a suitable level of hydration, and strive to consume 3 meals each day on a regular basis (emphasizing breakfast). Prior to discharge from the day treatment program, patients were informed of the possibility to participate in a new clinical trial, in which they could receive "medication alone" or "mindfulness training alone" (Med-Group or MTGroup).

Patients participating in the Med-Group received only prophylactic medications. The preventive compound was chosen on the basis of clinical history and medical comorbidities [49-52], such as done in routine care. Patients included in the MT-group, participated in a series of mindfulness training sessions and were not prescribed any form of prophylaxis. The mindfulness protocol we used was implemented on the basis of the Mindfulness-Based-Stress-Reduction MBSR program (MBSR) by Jon Zabat-Zinn [53]. Together with a close variant - the mindfulness-based cognitive therapy (MBCT) [54] - this is the most largely applied for various forms of recurrent pain [46], which we partially modified with regard to the frequency and the duration of sessions to increase the likelihood of adherence to treatment by patients. Training was provided in small groups (5-6 patients), that met in a relaxed and quiet room every consecutive Monday for 6 weekly sessions, each of about 45 min duration. All sessions were guided by an experienced neurologist trained in mindfulness practice. The order in which the different techniques and phases were administered- with the due caution and flexibility - was as follows. First, patients were provided a detailed explanation about the treatment protocol; i.e. what it is and what it is not, and in which clinical conditions it may be of most value. Second, patients were trained to assume a relaxed position that promoted good and regular breathing, while their eyes remained closed, with them maintaining a relaxed sitting position. Third, during the first meditations (approximately up to the second/third session), patients were invited to focus on attention on their breathing, on the present and on silence to enhance awareness of current mind and body sensations. Fourth, once patients learned to focus on the present, they erre requested to enhance awareness of their thoughts (third and fourth session), accepting them in a non-judgmental way. Fifth, in the last sessions (generally the last two), when patients had gathered higher awareness of their thoughts and the capacity to accept them, they were invited to preserve themselves from interfering thoughts, and to focus on the present and on the sensations they received from their bodies. When distractions occurred, patients were informed to resume attention to breathing and body awareness and observe the interfering content in a nonjudgmental way [55]. Finally patients were encouraged to supplement their training with regular home selfpractice, of 7-10 min per day (an amount typically recommended in studies of this type). The importance of practicing this form of meditation in an effortless 
manner was pointed out. Unlike prior investigations [41], we purposely limited the number of techniques to promote mastery of a few (versus exposure to numerous modalities where patients may be unable to master any to a meaningful degree) and keep time demands low and similar to that for pharmacological treatment.

This was an exploratory study, conducted in a working clinic that draws patients from a large coverage area, and the neurologist who guided the mindfulness sessions was one of the authors (LG), who had undergone extensive training in mindfulness at the Association for Meditation and Awareness under the supervision of Prof. Corrado Pensa. In this clinical setting it was not possible to implement random assignment, due to limited resources in terms of personnel and space. Another barrier lies in patients' provenance and past history: in fact, our center is a high-level specialty one, and patients seeking treatment come from all over the country, with some of them being followed-up for many years, thus making it difficult to enroll patients in a way that is strictly consistent with the requirements of a RCT. Therefore, certain aspects were not blinded: in particular, the investigators knew which patients attended mindfulness sessions and which not, as those that did not attend the sessions needed to be supervised with regard to eventual side effects of prophylactic medications. Participation was, thus, on a voluntary basis, with patients self-selecting their preferred treatment condition. Patients opting for mindfulness training alone were informed of the importance of being available to attend weekly sessions on a consistent basis upon discharge. Patients unable to commit to the stated schedule were invited to participate in the medical prophylaxis alone condition. Follow-up evaluations were carried out by another neurologist (DD) to limit possible source of bias with clinical outcomes, and questionnaire completion was supervised by a psychologist (ES). Given these considerations we view this investigation as more along the lines of an effectiveness trial (versus an efficacy trial) [56]. The consequence of this is that our results have to be taken as preliminary and the efficacy has to be tested in future randomized trials.

The study was approved by the Institute's Ethical Committee and written informed consent was obtained from each patient prior to enrollment in the study protocol. Follow-up assessments were conducted at 3, 6 and 12 months for all available patients. During this period, patients were instructed to continue their prior treatments. Patients in both groups were encouraged to restrict use of acute medications to headaches judged to be very disabling, operationally defined as a pain intensity rated as 8 or greater on a $0-10$ (no pain - pain as bad as it could be). Patients were instructed to take Eletriptan $(40 \mathrm{mg})$ and/or Almotriptan $(12.5 \mathrm{mg})$ as the first-line treatment, and indomethacin $(50 \mathrm{mg})$ as the second line; with regard to other NSAIDs, they were urged to take those medications that had already proved to be effective. Finally, in any case, they were strongly recommended to avoid opioids to the extent possible.

\section{Measures}

Headache diaries [25], completed on a daily basis, provided the primary measure of outcome, i.e., headache frequency, and the consumption of acute medications (NSAIDs and triptans). The amount of single intakes was recorded, irrespective of the kind of medication.

The Headache Impact Test (HIT-6) [57] is a 6-item scale that measures lost time in 3 domains and other areas of impact (e.g., pain severity, fatigue, and mood), based on patient recall for the immediate past 4 weeks. Each item is rated on a scale ranging from "never" to "always." Total scores range from 36 to 78 with higher scores indicating greater impact: scores $\geq 60$ are indicative of a severe impact.

The Migraine Disability Assessment (MIDAS) [58] is the most widely used measure of disability in headache research and we included it to facilitate comparisons with prior research. It is composed of 7 questions, all referenced to the preceding 3 months. The first 5 inquire about the number of days during which headache presence disrupted (partially or totally) paid and school work, household work, and leisure/family/social duties. Summing these individual values yields a total disability score, which correspond to four severity level: $0-5$, little or no disability; $6-10$, mild disability; $11-20$, moderate disability; 21 or above, severe disability. The remaining two items address the overall headache frequency and average pain intensity, measured on a $0-10$ scale. As headache frequency and intensity were prospectively obtained from daily diaries, and the validity of headache data recalled over three months is questionable [59], we elected to not report these data here.

Beck Depression Inventory (BDI) [60]. We used the 13-item version, which asks participants to rate the extent to which they are experiencing each of the 13 common symptoms of depression included. Items are rated on a scale from 0 to 3 (where 3 represents the highest severity), with the maximum score being 39 . When used as a screening device, a cut-off score of 9/10 seems best suited for indicating the presence/absence of depression [61].

The State-Trait Anxiety Inventory (STAI) Y1 and Y2 [62] is composed of two sections, each containing 20 items, that address state and trait anxiety, i.e., the transitory feelings that respondents experience in the moment in which they complete the questionnaire vs. the relatively stable and enduring personal features reflective of a predisposition to anxiety. Raw scores range between 
20 and 80 for each scale, with higher scores indicating higher anxiety levels. The raw scores can be transformed into norm-based T-Scores (mean 50, SD 10) to enable comparability across gender and age groups [63].

\section{Data analyses}

Mean baseline values for all demographic and dependent variables for the 2 treatment conditions were compared by $t$-tests. The primary and secondary outcome variables were analyzed in separate two-way mixed ANOVAs (Group: Mindfulness vs. Pharmacology x Time: Baseline, 3-, 6-, vs. 12-month follow-up), followed by post-hoc tests, with appropriate adjustments made when significant effects were obtained in order to guard against inflation of the familywise error rate. Partial eta squared $\left(\eta^{2}\right)$ values were calculated for all significant findings, conservatively interpreting them as small (.01-.08), medium (.09-.24), and large ( $\geq .25)$.

We additionally evaluated clinical significance by determining the percentage of patients that, compared to baseline evaluation, achieved a $50 \%$ or greater reduction in migraine frequency and the percentage of patients who no longer met the diagnostic criteria for $\mathrm{CM}$ at each of the three follow-up evaluations. We then compared the ratios between the two groups of patient (MT-Group and Med-Group), for each of these 2 additional measures, at each time-point using Chi-Squared analyses. The $p$-value for significance for all tests was set at .05 .

\section{Results}

Fifty patients met inclusion criteria during the study period, but six declined to participate due to lack of time or interest. Forty-four patients were therefore enrolled in this trial, 22 in each condition. In the Med-Group, five patients received valproate, eight botulinum toxin, five pizotifen, one amitriptyline, two received a combination of beta blockers and amitriptyline and one was given beta blockers and valproate. Table 1 reports the mean baseline values for all enrolled patients, collectively and by group assignment, along with current age and age at onset of headache. No differences were found between the 2 groups for any measures (the same was true when comparing baseline values for those who completed the trial; $n=39$ versus those who did not, $n=3$ ). At baseline, a similar percentage of patients were overusing triptans: 8 of 22 patients $(36.4 \%)$ in the Med-Group and 6 of 22 patients (27.3\%) in the MT-Group $\left(\mathrm{X}^{2}(1)=0.42, p=.52\right)$. The 2 groups did differ with respect to overuse of NSAIDS: 22 of $22(100 \%)$ for Med-Group vs. 18 of 22 for MT-Group $\left(\mathrm{X}^{2}(1)=4.40, p=.04\right)$.

Figure 1 shows the flow of patients in each of the two groups: complete follow-up data at 12 months was available for 19 patients in the Med-Group and for 20 in the MT-Group.

\section{Primary and secondary outcomes}

Mean values for all measures at all time points are reported for patients who completed the entire trial (see Table 2). Four of the seven separate mixed withinbetween subjects ANOVAs revealed significance only for the main effect of time- Headache Frequency, Medication intake, MIDAS, and BDI-13 (see Table 3). Analysis of the HIT- 6 data revealed not only a main effect for time, but also for the interaction of time and group. Neither analysis for STAI-S or STAI-T revealed any significant effects (so these variables are not discussed further here). Pair wise post-hoc comparisons with Bonferroni corrections revealed all 3 follow-up points as significantly improved with respect to baseline values, but no differences among the 3 follow-up periods, for Headache Frequency, Medication intake, and BDI-13. MIDAS scores were significantly different from baseline

Table 1 Mean (and SD) baseline values for all measures for patients who began the trial, all 44 combined and separately for condition assignment, and statistical comparisons among the groups

\begin{tabular}{|c|c|c|c|c|c|c|c|c|}
\hline \multirow[t]{2}{*}{ Variable } & \multicolumn{2}{|c|}{$\begin{array}{l}\text { Total } \\
(N=44)\end{array}$} & \multicolumn{2}{|c|}{$\begin{array}{l}\text { MT-Group } \\
(N=22)\end{array}$} & \multicolumn{2}{|c|}{$\begin{array}{l}\text { Med-Group } \\
(N=22)\end{array}$} & \multirow[t]{2}{*}{$t(42)$} & \multirow[t]{2}{*}{$P$} \\
\hline & M & $S D$ & M & SD & M & SD & & \\
\hline Age & 44.5 & 9.2 & 45.6 & 9.3 & 43.5 & 9.2 & 0.75 & .457 \\
\hline Age at Onset & 20.4 & 9.0 & 21.5 & 10.5 & 19.3 & 7.3 & 0.80 & .428 \\
\hline Headache frequency/month & 20.5 & 7.9 & 19.2 & 7.8 & 21.9 & 7.8 & -1.14 & .263 \\
\hline Monthly medication intake & 18.4 & 6.5 & 18.0 & 6.4 & 18.8 & 6.7 & -0.41 & .681 \\
\hline HIT & 66.3 & 5.2 & 65.5 & 5.5 & 67.1 & 4.9 & -1.05 & .301 \\
\hline MIDAS & 73.1 & 39.9 & 65.3 & 41.4 & 81.0 & 37.6 & -1.32 & .194 \\
\hline BDI-13 & 13.3 & 6.1 & 13.1 & 5.8 & 13.6 & 6.6 & -0.32 & .754 \\
\hline STAI-S & 48.2 & 7.3 & 47.1 & 6.6 & 49.4 & 7.9 & -1.06 & .297 \\
\hline STAI-T & 52.3 & 9.6 & 52.1 & 9.3 & 52.5 & 10.0 & -0.13 & .901 \\
\hline
\end{tabular}




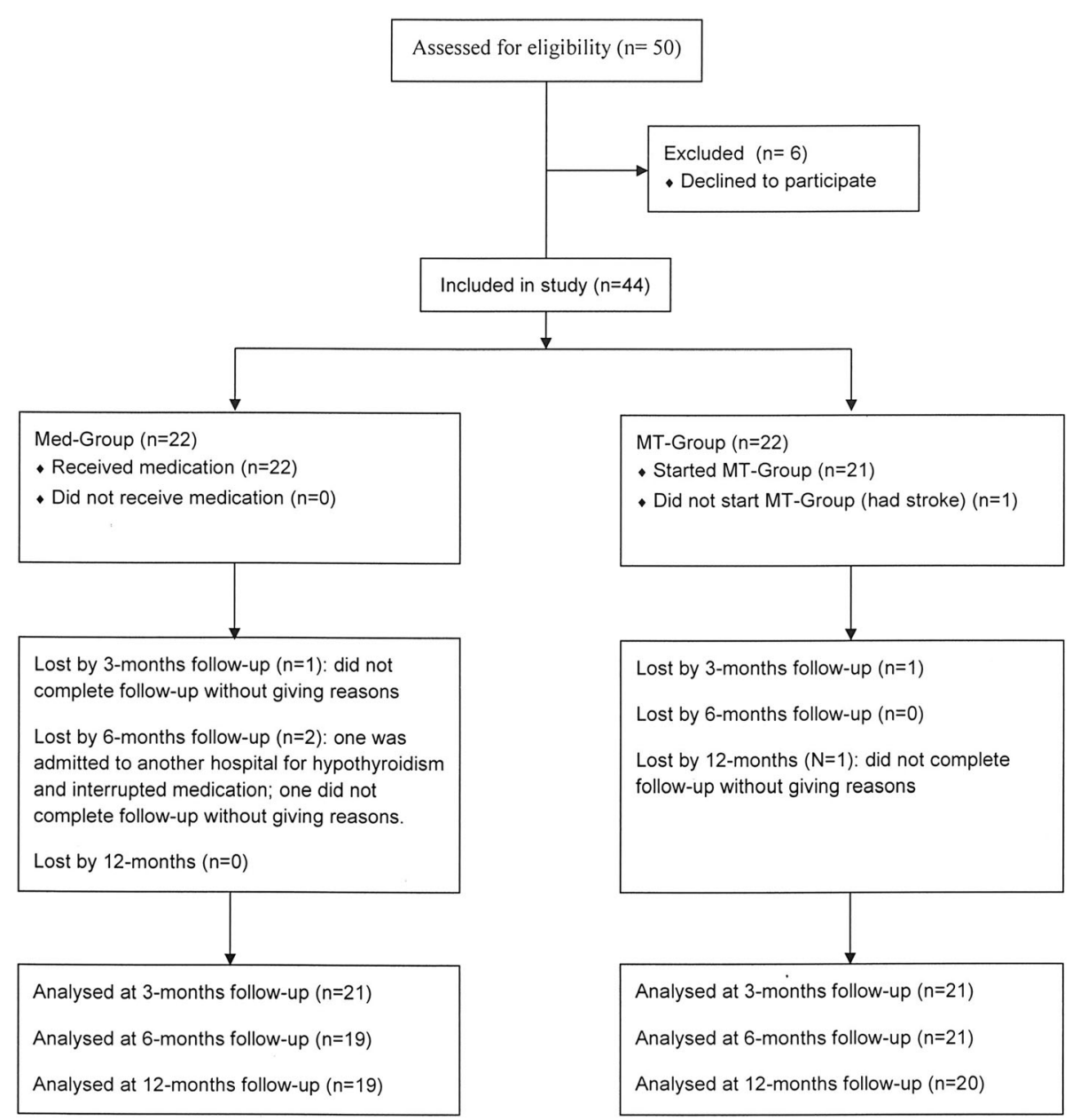

Fig. 1 Flowchart of the study

for the 3- and 6-month follow-up, but not for the 12month follow-up (see Table 4). Figure 2 graphically presents the outcomes for headache frequency and consumption of medication for management of acute headaches over the 12-month follow-up period.

To investigate the source of the significant interaction effect for the HIT-6, we conducted all pairwise comparisons for all time points for each group separately (see Table 5 and Fig. 3). Pairwise comparisons, with Sidak correction, revealed no differences between any time points for the MF-Group. For the MED-Group the 3 -month and 12-month values were significantly reduced when compared to baseline. No other differences emerged.

Table 2 Mean values and SD's for mindfulness (MT-Group) and pharmacology (MED-Group) at each measurement period

\begin{tabular}{|c|c|c|c|c|c|c|c|c|}
\hline & \multicolumn{4}{|l|}{ MT-Group } & \multicolumn{4}{|l|}{ Med-Group } \\
\hline & Baseline & 3-MO & $6-\mathrm{MO}$ & $12-\mathrm{MO}$ & Baseline & 3-MO & $6-\mathrm{MO}$ & $12-\mathrm{MO}$ \\
\hline Headaches Frequency & $18.5 \pm 7.2$ & $8.3 \pm 3.5$ & $10.4 \pm 6.9$ & $12.4 \pm 8.5$ & $18.5 \pm 7.2$ & $8.9 \pm 8.0$ & $11.4 \pm 8.0$ & $10.4 \pm 7.2$ \\
\hline Medications intake & $17.7 \pm 5.9$ & $8.1 \pm 4.6$ & $8.9 \pm 4.2$ & $10.3 \pm 5.4$ & $15.4 \pm 4.4$ & $8.8 \pm 8.4$ & $11.0 \pm 7.6$ & $8.6 \pm 4.8$ \\
\hline HIT-6 & $65.3 \pm 5.7$ & $62.0 \pm 5.7$ & $60.7 \pm 10.8$ & $64.5 \pm 7.0$ & $66.9 \pm 5.2$ & $60.7 \pm 7.7$ & $62.6 \pm 6.3$ & $61.5 \pm 4.8$ \\
\hline MIDAS & $65.4 \pm 43.5$ & $39.0 \pm 36.7$ & $41.5 \pm 51.7$ & $53.7 \pm 52.6$ & $82.9 \pm 40.0$ & $26.7 \pm 23.5$ & $38.8 \pm 25.4$ & $51.5 \pm 50.2$ \\
\hline BDI-13 & $13.4 \pm 5.9$ & $9.0 \pm 6.3$ & $9.0 \pm 5.3$ & $10.3 \pm 6.8$ & $13.3 \pm 6.8$ & $6.2 \pm 6.3$ & $8.0 \pm 5.9$ & $7.6 \pm 6.4$ \\
\hline STAI-T & $52.3 \pm 9.8$ & $48.6 \pm 8.4$ & $48.6 \pm 7.7$ & $50.9 \pm 9.5$ & $52.8 \pm 10.2$ & $48.5 \pm 9.4$ & $51.3 \pm 9.9$ & $48.4 \pm 9.6$ \\
\hline STAI-S & $47.0 \pm 6.8$ & $45.4 \pm 6.4$ & $45.5 \pm 6.9$ & $49.9 \pm 9.3$ & $49.6 \pm 7.8$ & $47.2 \pm 6.1$ & $48.3 \pm 10.7$ & $48.6 \pm 8.7$ \\
\hline
\end{tabular}

$N=19$ for MT-Group and $N=20$ for MED-Group

Note. Values are expressed as means $\pm \mathrm{SD}$ 
Table 3 Mixed within-between ANOVA results

\begin{tabular}{|c|c|c|c|c|c|c|c|c|}
\hline \multirow[t]{2}{*}{ Variable } & \multicolumn{3}{|c|}{ Main effects for time } & \multicolumn{2}{|c|}{ Main effect for group } & \multicolumn{3}{|c|}{ Interaction (Time X Group) } \\
\hline & Wilks' lambda & $P$ & partial $\eta^{2}$ & $F$ & $p$ & Wilks' lambda & $p$ & partial $\eta^{2}$ \\
\hline Headaches frequency & .43 & $<.001$ & .57 & 0.00 & .959 & .93 & .453 & .07 \\
\hline Medications intake & .31 & $<.001$ & .69 & 0.04 & .842 & .83 & .094 & .17 \\
\hline HIT & .67 & .002 & .34 & 0.02 & .902 & .76 & .020 & .24 \\
\hline MIDAS & .43 & $<.001$ & .57 & 0.02 & .902 & .86 & .141 & .14 \\
\hline BDI-13 & .49 & $<.001$ & .51 & 1.12 & .297 & .93 & .475 & .07 \\
\hline STAI-S & .82 & .064 & .19 & 0.70 & .408 & .93 & .481 & .07 \\
\hline STAI-T & .82 & .068 & .18 & 0.00 & .952 & .89 & .246 & .11 \\
\hline
\end{tabular}

Percent improvement and percent of patients no longer meeting criteria for $\mathrm{CM}$

Table 6 reports the percentage of patients showing 50\% or more reduction of headaches compared to baseline and of patients no longer meeting criteria for CM for each of the time-points. For both of the clinical endpoints, there were no differences between patients in the MT-Group and those in the Med-Group. With regard to the percent improvement variable, the trend had a $\mathrm{U}$ shaped curve in both groups, while the trend had a Jshaped curve with regard to the number of patients no longer meeting the criteria for CM.

Table 4 Post-hoc comparisons across time for significant time main effects

\begin{tabular}{|c|c|c|c|c|}
\hline Variable & M & $S D$ & $M$ diff & $P$ \\
\hline \multicolumn{5}{|l|}{ Freq } \\
\hline Baseline (ref) & 18.5 & 7.1 & & \\
\hline 3-month & 8.6 & 6.1 & 9.9 & $<.001$ \\
\hline 6-month & 10.9 & 7.4 & 7.6 & $<.001$ \\
\hline 12-month & 11.4 & 7.9 & 7.1 & $<.001$ \\
\hline \multicolumn{5}{|c|}{ Medications intake } \\
\hline Baseline (ref) & 16.6 & 5.3 & & \\
\hline 3-month & 8.4 & 6.6 & 8.2 & $<.001$ \\
\hline 6-month & 9.9 & 6.1 & 6.7 & $<.001$ \\
\hline 12-month & 9.5 & 5.1 & 7.1 & $<.001$ \\
\hline \multicolumn{5}{|l|}{ MIDAS } \\
\hline Baseline (ref) & 73.9 & 42.2 & & \\
\hline 3-month & 33.0 & 31.2 & 40.9 & $<.001$ \\
\hline 6-month & 40.2 & 40.6 & 33.7 & $<.001$ \\
\hline 12-month & 52.6 & 50.8 & 21.3 & ns \\
\hline \multicolumn{5}{|l|}{ BDI-13 } \\
\hline Baseline (ref) & 13.3 & 6.2 & & \\
\hline 3-month & 7.6 & 6.4 & 5.7 & $<.001$ \\
\hline 6-month & 8.5 & 5.5 & 4.9 & $<.001$ \\
\hline 12-month & 9.0 & 6.7 & 4.4 & .001 \\
\hline
\end{tabular}

Note: Only comparisons to baseline scores are presented in this table, as all other pairwise comparisons were not statistically significant

\section{Discussion}

Our preliminary data show that both groups of patients, treated with only a single, non-combination intervention-conventional pharmacological approach only versus a mindfulness-based approach only-revealed significant decreases in number of monthly headache days, monthly consumption of medication for acute headache management, MIDAS, and depressive symptoms up to 12-months follow-up. The change in mean BDI-13 scores (collapsing across groups), appears to be of clinical significance, as the baseline values, which fell into the stringent range (13/14) for tentatively identifying moderate to severe clinical depression, had by 12 months fallen overall within the lowest end of the range suggesting the possible presence of moderate/severe depression (9/10). Although MIDAS scores decreased from baseline by $28.8 \%$ at 12 months, the mean score at this time point (averaged across both conditions) continued to fall within the highest severity grade Level (IV). Headache impact was reduced to a statistically significant degree at 3- and 12-month follow-up, but only for the MED-Group. It is important to point out that at all time points, neither group revealed clinically meaningful reductions on this measure because means continued to fall within the highest severity category for this scale (all means $\geq 60$ ). Given the long-standing duration of chronic headache activity by our patients, it is not surprising that headache impact and depression did not reveal more marked changes, even at 1 year follow-up. Changes of a psychological nature often take additional time to fully manifest [26]. Anxiety scores remained unchanged over time. Although anxiety disorders and CM are known to be comorbid [64] - STAI scores revealed the absence of significant anxiety problems in either group, thus leaving little room for change ("basement" or floor effect).

The proportion of patients achieving a 50\% reduction in headaches frequency (a measure commonly used to evaluate "clinically significant improvements") was similar at all time-points (at 12 months: 50\% in the MTGroup, $52.6 \%$ in the Med-Group), and the same was true 


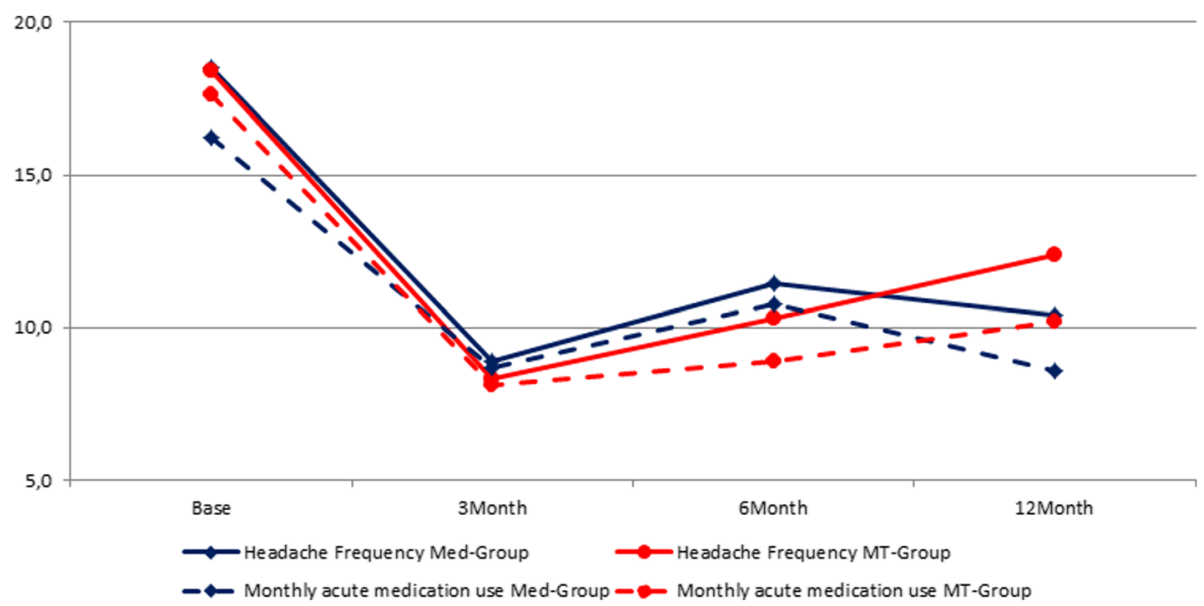

Fig. 2 Longitudinal course of headache frequency and consumption of medication. Note. Differences were always significant compared to baseline; no differences were found between the Med-Group and the MT-Group at any time point

for the proportion of patients no longer meeting the $\mathrm{CM}$ criteria (at 12 months: 65\% in the MT-Group, $73.7 \%$ in the Med-Group). Taken as a whole, our results suggest that the longitudinal course of patients receiving Mindfulness-based treatment, and who were instructed to refrain from medical prophylaxis (which was verified by the dairy records participants maintained throughout the study), was overall very similar to that for patients who were administered conventional medical prophylaxis, with few exceptions noted.

Our preliminary data extend the findings of Wells and colleagues [40], whose mindfulness intervention was focused on episodic migraine and reported more limited findings. To our knowledge, only one previous study has examined the utility of mindfulness-based treatments for chronic forms of headache (CM or Tension-Type Headache, with the distribution not being reported) wherein mindfulness was also examined as an "add-on" therapy and consisted of a host of other therapeutic components, some derived from a mindfulness framework but many derived from other theoretical models [41]. These investigators found significant differences between MBSR + pharmacoterapy and pharmacotherapy alone with respect to perceived pain intensity and quality of life. However, it is not clear if their treatment impacted headache frequency, which is our primary measure and the primary outcome recommended by the most recent IHS clinical trial guidelines [65], or other outcome measures such as those that we included, i.e., use of acute medications, disability burden and mood. The fact that positive effects (although not always reaching significant changes) were observed in our study for these varied measures in patients who received our brief mindfulness training alone (in the absence of prophylactic medications)

Table 5 Pairwise comparisons between time points of HIT for each treatment group

\begin{tabular}{|c|c|c|c|c|c|c|c|}
\hline \multirow[t]{2}{*}{ Group } & \multicolumn{2}{|c|}{ Comparisons } & \multirow{2}{*}{$\begin{array}{l}\text { Mean difference } \\
\text { (A-B) }\end{array}$} & \multirow[t]{2}{*}{ SE } & \multirow[t]{2}{*}{$p$} & \multicolumn{2}{|c|}{$95 \% \mathrm{Cl}$ of difference } \\
\hline & Time A & Time B & & & & $\overline{L L}$ & UL \\
\hline \multirow[t]{6}{*}{ Mindfulness } & Baseline & $3 M$ & 3.25 & 1.56 & .240 & -1.10 & 7.60 \\
\hline & & $6 \mathrm{M}$ & 4.55 & 2.21 & .247 & -1.58 & 10.68 \\
\hline & & $12 \mathrm{M}$ & 0.80 & 1.73 & .998 & -4.00 & 5.60 \\
\hline & $3 M$ & $6 \mathrm{M}$ & 1.30 & 1.87 & .983 & -3.91 & 6.51 \\
\hline & & $12 M$ & -2.45 & 1.81 & .705 & -7.48 & 2.58 \\
\hline & $6 \mathrm{M}$ & $12 \mathrm{M}$ & -3.75 & 1.43 & .073 & -7.73 & 0.23 \\
\hline \multirow[t]{6}{*}{ Pharmacological } & Baseline & $3 M$ & 6.21 & 1.60 & .003 & 1.75 & 10.67 \\
\hline & & $6 \mathrm{M}$ & 4.32 & 2.26 & .328 & -1.97 & 10.60 \\
\hline & & $12 \mathrm{M}$ & 5.42 & 1.77 & .024 & 0.50 & 10.34 \\
\hline & $3 M$ & $6 M$ & -1.90 & 1.92 & .910 & -7.24 & 3.45 \\
\hline & & $12 \mathrm{M}$ & -0.79 & 1.86 & .999 & -5.95 & 4.37 \\
\hline & $6 M$ & $12 \mathrm{M}$ & 1.11 & 1.47 & .974 & -2.97 & 5.18 \\
\hline
\end{tabular}




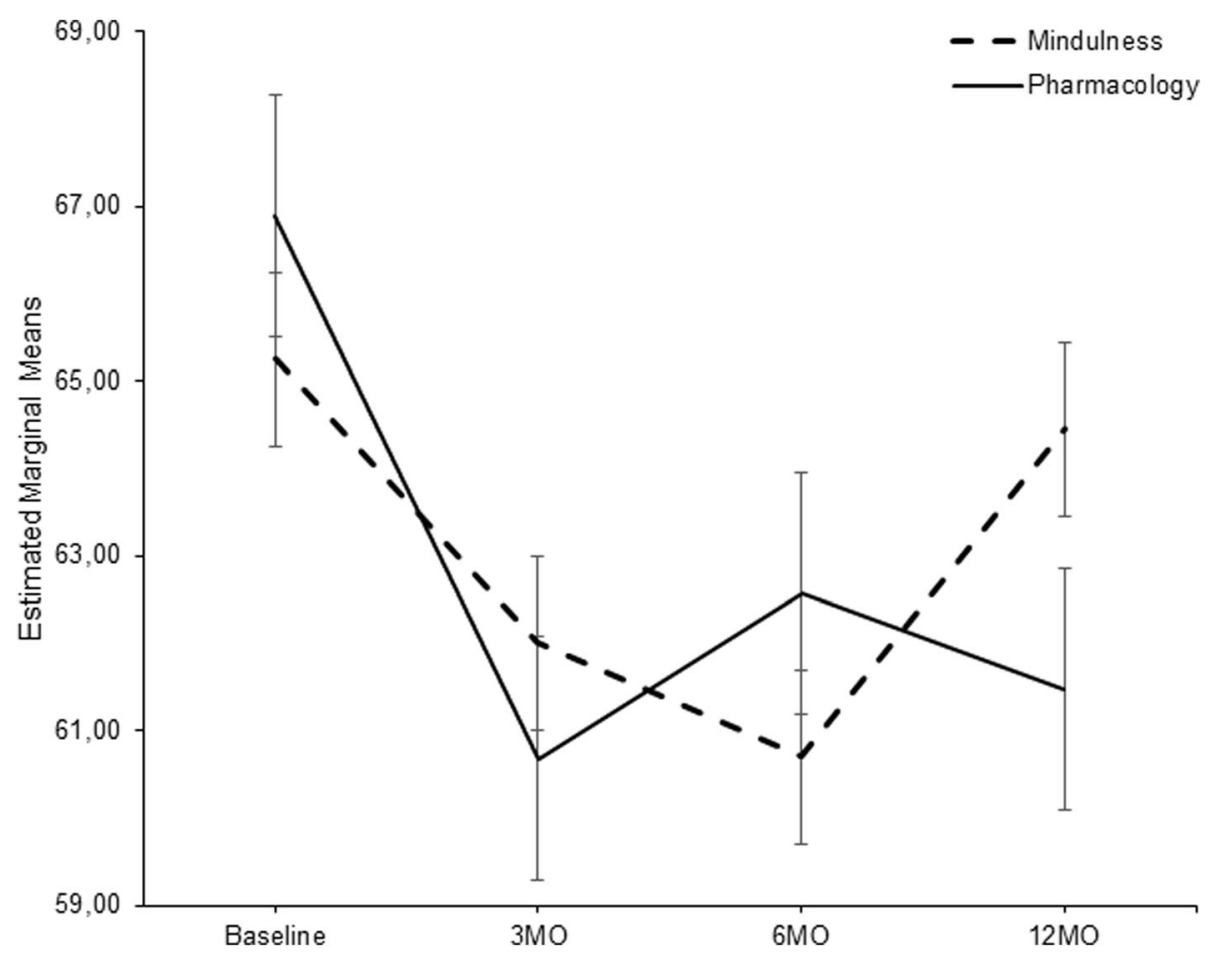

Fig. 3 Estimated marginal means for HIT-6 scores across time for Mindfulness and Pharmacology groups. Error bars represent standard errors

suggests that mindfulness-based treatment may be comparable to standard pharmacological prophylaxis as far as its global positive clinical improvement. However, the absence of random assignment and the fact that our study was not cast as a non-inferiority trial leaves this possibility more as a hypothesis, one in need of further testing.

Mood is one of the most important non-headache factors associated with migraine chronification [7, 64] and reducing headache frequency can lead to reductions in depression [29, 66]. Nonetheless, the relevance of depressive symptoms remains somewhat controversial. In fact, in previous studies on CM-MO samples [24, 67] depression scores - measured with the updated BDI-II [68] and not with the original BDI - were not correlated with frequency of headaches and, when implemented in a predictive model together with headache frequency

Table 6 Percent improvement and percent of patients no longer meeting criteria for CM at each time-point

\begin{tabular}{lllll}
\hline & & MT-Group & Med-Group & $\begin{array}{l}\text { Chi-Squared } \\
(P \text {-value })\end{array}$ \\
\hline 50\% reduction & 3 Months & $57.1 \%$ & $76.2 \%$ & $1.71(P=.190)$ \\
& 6 Months & $47.6 \%$ & $26.3 \%$ & $1.93(P=.165)$ \\
& 12 Months & $50.0 \%$ & $52.6 \%$ & $0.03(P=.869)$ \\
No longer CM & 3 Months & $95.2 \%$ & $90.0 \%$ & $0.41(P=.520)$ \\
& 6 Months & $76.2 \%$ & $78.9 \%$ & $0.04(P=.835)$ \\
& 12 Months & $65.0 \%$ & $73.7 \%$ & $0.35(P=.557)$ \\
\hline
\end{tabular}

and pain intensity, BDI-II scores had higher value in predicting disability and quality of life scores. Our finding that Mindfulness practice (as well as medication) had a modest positive effect on levels of reported depression over time is compatible with a conclusion previously drawn in studies addressing depression and mindfulness-based treatments [69-73]. These findings show that the effect of mindfulness-based approaches on symptoms of depression were superior to psychoeducational intervention and non-inferior to individual cognitive behavioral therapy, that they yielded similar results compared to antidepressant therapies and, finally, that the effect is maximized when the treatment is combined. Considering that only $40 \%$ of patients in the MED-group received a therapy having some kind of mood-modulating effect, the finding that the impact on mood component was similar is in line with the previous report, and suggests that mindfulness-based treatments, combined with appropriate antidepressant therapy, might yield an increased impact on symptoms of depression.

Although mean BDI values for both groups were in the range of a significant levels of depression prior to treatment, we hesitate to speculate further about the meaningfulness of the changes reported here given that our measure of depression is intended primarily as a screening instrument and does not take the place of a careful clinical diagnosis. 
More recent conceptions, wherein migraine is being recognized as a condition in which biological, social and psychological aspects are very interconnected [25] has helped to increase awareness of the need to modify therapeutic approaches to include newer and sometimes "non-conventional" options (behaviorally and cognitively based), along with "traditional" treatments (i.e., medical) to better help patients to manage their condition, reduce their medication intake, and minimize the incidences of relapse in overuse after withdrawal $[25,74]$.

Mindfulness is designed to promote the ability to focus on and accept the present situations and the difficulties of every day. As demonstrated by Kabat-Zinn [75], patients who have been educated to use mindfulness may better manage stressful situations, increase their self-efficacy, and learn to manage pain more adequately avoiding the compulsion between pain and medication intake which easily sets in motion the vicious cycle of pain and medication and to the condition of overuse. Mindfulness research, especially as regards headache, remains in the infancy stage, with many aspects in need of further investigation. Among these are determining which of the myriad of bio-psycho-social factors may underlie treatment effectiveness, including, for example, changes in perception of and reactions to pain sensations and emotion, self-efficacy and coping abilities, physiology, cerebral structures and circuits [19, 38, 46, 47].

Although our findings are encouraging and suggestive of the independent value of mindfulness for headache care, certain design limitations preclude us making unequivocal claims. Our inability to randomize patients to conditions serves as a limiting factor, with results perhaps applying only to those particularly motivated to commit to an extended training period for mindfulness. Our headache center is designed primarily as a fee for service clinic, where patient preferences must be considered. However, as pointed out by Nash et al [56] trials of this type, more aptly termed "effectiveness trials" (versus the more standard "efficacy trials") clearly have a place in the early stage of treatment development. In this case, we hope our findings serve to expand recently published data on patients with primary headaches supporting the clinical value of mindfulness in the most severely affected patients in the migraine spectrum; i.e., those with chronic migraine coupled with medication overuse. Another consideration is our inability to document the extent to which patients adhered to each treatment (no dose monitoring for the MED group and no checks for amount of mindfulness practice or the depth of learning patients acquired). Nonetheless, we believe our findings support the value of conducting further more wellcontrolled studies (incorporating random assignment, larger samples sizes, and checks on integrity of treatment) are warranted to more fully explore the benefits, boundaries, and mechanisms of action for mindfulness in treating chronic migraine by itself and when it is complicated by medication overuse and medical or psychological comorbidities. Finally, our sample was composed of patients with $\mathrm{CM}$ and with no psychiatric comorbidities. Two literature reviews showed that migraineurs, compared to the general population, are much more likely to suffer from psychiatric comorbidities (up to $60-70 \%$ for mood and anxiety disorders) and that women with migraine with aura are at an increased risk of suicide attempt [76, 77]. Conversely, the evidence on the relationships between mood disorders expression and suicidal ideation seems contrasting, with some studies finding and others not finding any connections [78, 79]. Patients included in our sample seem to be clearly different from those described in these previous studies, as they did not have psychiatric comorbidity - although some degree of low mood was found - and actually we had no reasonable ground to suspect any suicidal ideation among the participants herein included. Caution is therefore recommended before generalizing our results to the entire population of $\mathrm{CM}$ patients: for all of the above reasons, our results should be taken as preliminary.

\section{Conclusions}

Our results provide initial support for the beneficial effect of Mindfulness-based treatment in the management of chronic migraine that is accompanied with medication overuse, a headache form which represents a clinical challenge. Our results further suggest that a Mindfulness-based treatment may be comparable to standard pharmacological prophylaxis with regard to relevant primary outcomes such as headaches frequency reduction and reduction in the consumption of acute medications.

\section{Abbreviations}

BDI: Beck depression inventory; CM: Chronic migraine; CM-MO: Chronic migraine associated with medication overuse; HIT-6: Headache impact test; ICHD-3Beta: International Classification of Headache Disorder III edition, beta version; MBCT: Mindfulness-based cognitive therapy; MBSR: Mindfulness based stress reduction; Med-Group: Medication alone group; MIDAS: Migraine disability assessment; MO: Medication overuse; MT-Group: Mindfulness training alone group; NSAIDs: Non-steroidal anti-inflammatory drugs; STAI: State-trait anxiety inventory; TAU: Therapy as usual; TTH: Tension-type headache

\section{Funding}

The study was supported by the Neurological Institute C. Besta IRCCS Foundation and by the European Commission, Seventh Framework Programme, Grant No. 316795 (MARATONE).

\section{Authors' contributions}

LG - Enrolment of patients, MT administration, prescription of prophylaxis. ES - Enrolment of patients, outcome measures collection, revision of the manuscript. AR - Preparation of the first draft of the manuscript and data analysis. DD - Selection and follow-up of patients, revision of the entire manuscript. ADG - Preparation of the protocol. ML - Supervision of the manuscript. LDT - Supervision of the manuscript. FSG - Data analysis. FA - Revision of the entire manuscript and data analysis. All authors read and approved the final manuscript. 


\section{Competing interests}

The authors declare that they have no competing interests.

\section{Ethics approval and consent to participate}

The study was approved by the Institute's ethics committee, and was therefore been performed in accordance with the ethical standards laid down in the 1964 Declaration of Helsinki and its later amendments.

\section{Author details}

'Neurological Institute "C. Besta" IRCCS Foundation, Headache and Neuroalgology Unit, Via Celoria 11, 20133 Milan, Italy. ${ }^{2}$ Neurological Institute "C. Besta" IRCCS Foundation, Neurology, Public Health and Disability Unit, Milan, Italy. ${ }^{3}$ eCampus University, Faculty of Psychology, Novedrate, Italy. ${ }^{4}$ Department of Psychology, Univeristy of Memphis, Memphis, TN, USA.

\section{Received: 6 December 2016 Accepted: 27 January 2017}

\section{Published online: 04 February 2017}

\section{References}

1. Global Burden of Disease Study 2013 Collaborators (2015) Global, regional, and national incidence, prevalence, and years lived with disability for 301 acute and chronic diseases and injuries in 188 countries, 1990-2013: a systematic analysis for the Global Burden of Disease Study 2013. Lancet 386: 743-800

2. Steiner TJ, Stovner LJ, Vos T (2016) GBD 2015: migraine is the third cause of disability in under 50s. J Headache Pain 17:104

3. Manack AN, Buse DC, Lipton RB (2011) Chronic migraine: Epidemiology and disease burden. Curr Pain Headache Rep 15:70-78

4. Giannini G, Favoni V, Bauleo S, Ferrante T, Pierangeli G, Albani F, Bacchi Reggiani ML, Baruzzi A, Cortelli P, Cevoli S (2012) SPARTACUS: underdiagnosis of chronic daily headache in primary care. Neurol Sci 33:181-183

5. Headache Classification Committee of the International Headache Society (2013) The International Classification of Headache Disorders, 3rd edition (beta version). Cephalalgia 33:629-808

6. Cevoli S, Sancisi E, Grimaldi D, Pierangeli G, Zanigni S, Nicodemo M, Cortelli P, Montagna P (2009) Family history for chronic headache and drug overuse as a risk factor for headache chronification. Headache 49:412-418

7. Bigal M (2009) Migraine chronification-concept and risk factors. Discov Med 8:145-150

8. Thorlund K, Sun-Edelstein C, Druyts E, Kanters S, Ebrahim S, Bhambri R, Ramos E, Mills EJ, Lanteri-Minet M, Tepper S (2016) Risk of medication overuse headache across classes of treatments for acute migraine. $J$ Headache Pain 17:107

9. Andrasik F, Grazzi L, Usai S, Buse DC, Bussone G (2009) Non-pharmacological approaches to treating chronic migraine with medication overuse. Neurol Sci 30:89

10. Diener HC, Dodick DW, Goadsby PJ (2009) Utility of topiramate for the treatment of patients with chronic migraine in the presence or absence of acute medication overuse. Cephalalgia 29:1021-1027

11. Aurora SK, Dodick DW, Turkel CC, DeGryse RE, Silberstein SD, Lipton RB (2010) OnabotulinumtoxinA for treatment of chronic migraine: Results from the double-blind, randomized placebo-controlled phase of the PREEMPT 1 trial. Cephalalgia 30:793-803

12. D'Amico D, Grazzi L, Usai S, Raggi A, Leonardi M, Bussone G (2011) Disability in chronic daily headache: state of the art and future directions. Neurol Sci 32:S71-S76

13. Evers $S$, Jensen $R$ (2011) Treatment of medication overuse headacheGuideline of the EFNS headache panel. Eur J Neurol 18:1115-1121

14. Raggi A, Giovannetti AM, Leonardi M, Sansone E, Schiavolin S, Curone M, Grazzi L, Usai S, D'Amico D (2017) Predictors of 12-Months Relapse After Withdrawal Treatment in Hospitalized Patients With Chronic Migraine Associated With Medication Overuse: A Longitudinal Observational Study. Headache 57:60-70

15. Biagianti B, Grazzi L, Usai S, Gambini O (2014) Dependency-like behaviors and pain coping styles in subjects with chronic migraine and medication overuse: Results from a 1-year follow-up study. BMC Neurol 14:181

16. Rossi P, Faroni JV, Nappi G (2008) Medication overuse headache: Predictors and rates of relapse in migraine patients with low medical needs. A 1-year prospective study. Cephalalgia 28:1196-1200
17. Grazzi L, Andrasik F, Usai S, Bussone G (2009) Treatment of chronic migraine with medication overuse. Is drug withdrawal crucial? Neurol Sci 30:S85-S88

18. Rossi P, Faroni JV, Nappi G (2011) Short-term effectiveness of simple advice as a withdrawal strategy in simple and complicated medication overuse headache. Eur J Neurol 18:396-401

19. Candy R, Farmer K, Beach ME, Tarrasch J (2008) Nurse-based education: an office-based comparative model for education of migraine patients. Headache 48:564-569

20. Grazzi L (2008) Behavioural approach to the difficult patient. Neurol Sci 29: \$96-\$98

21. Andrasik F, Grazzi L, Usai S, Kass S, Bussone G (2009) Disability in chronic migraine with medication overuse: Treatment effects through 5 years. Cephalalgia 30:610-614

22. Holroyd KA, Drew JB, Cottrell CK, Romanek KM, Heh V (2007) Impaired functioning and quality of life in severe migraine: The role of catastrophizing and associated symptoms. Cephalalgia 27:1156-1165

23. Wang SJ, Wang PJ, Fuh JL, Penh KP, Ng K (2013) Comparisons of disability, quality of life, and resource use between chronic and episodic migraineurs: A clinic based study in Taiwan. Cephalalgia 33:171-181

24. Raggi A, Curone M, Schiavolin S, Di Fiore P, Leonardi M, Grazzi L (2015) Chronic migraine with medication overuse: Association between disability and quality of life measures, and impact of disease on patients' lives. J Neurol Sci 348:60-66

25. Andrasik F, Flor H, Turk DC (2005) An expanded view of psychological aspects in head pain: the biopsychosocial model. Neurol Sci 26:S87-S89

26. Grazzi L, Andrasik F, D'Amico D, Leone M, Usai S, Kass SJ (2002) Behavioral and pharmacologic treatment of transformed migraine with analgesic overuse: Outcome at 3 years. Headache 42:483-490

27. Rains JC, Penzien DB, Lipchik GL (2006) Behavioral facilitation of medical treatment for headache-part II. Theor Models Behav Strategies Improv Adherence Headache 46:1395-1403

28. Andrasik F (2007) What does the evidence show? Efficacy of behavioral treatments for recurrent headache in adults. Neurol Sci 28:S70-\$77

29. Nestoriuc Y, Martin A, Rief W, Andrasik F (2008) Biofeedback Treatment for Headache Disorders: A Comprehensive Efficacy Review. Appl Psychophysiol Biofeedback 33:125-140

30. Rausa M, Palomba D, Cevoli S, Lazzerini L, Sancisi E, Cortelli $P$, Pierangeli G (2016) Biofeedback in the prophylactic treatment of medication overuse headache: a pilot randomized controlled trial. J Headache Pain 17:87

31. Rosenzweig S, Greeson JM, Reibel DK, Jasser SA, Beasley D (2010) Mindfulness based stress reduction for chronic pain condition: Variation in treatment outcomes and role of home meditation practice. J Psychosom Res 68:29-36

32. Chiesa A, Serretti A (2010) Systematic review of neurobiological and clinical features of mindfulness meditations. Psychol Med 40:1239-1252

33. Abdollah O, Fatemeh Z (2014) Effect of mindfulness-based stress reduction on pain severity and mindful awareness in patients with tension headache: a randomized controlled clinical trial. Nurs Midwifery Stud 3:e21136

34. Makenzie E, Wachholtz A (2014) Meditation Based Treatment Yielding Immediate Relief for Meditation-Naïve Migraineurs. Pain Manag Nurs 15:36-40

35. McCracken LM, Gauntlett-Gilbert J, Vowles KE (2007) The role of mindfulness in a contextual cognitive-behavioral analysis of chronic pain-related suffering and disability. Pain 131:63-69

36. Morone NE, Greco CM, Weiner DK (2008) Mindfulness meditation for the treatment of chronic low back pain in older adults: a randomized controlled pilot study. Pain 134:310-319

37. Castelnuovo G, Giusti EM, Manzoni GM, Saviola D, Gatti A, Gabrielli S, Lacerenza M, Pietrabissa G, Cattivelli R, Spatola CA, Corti S, Novelli M, Villa V, Cottini A, Lai C, Pagnini F, Castelli L, Tavola M, Torta R, Arreghini M, Zanini L, Brunani A, Capodaglio P, D'Aniello GE, Scarpina F, Brioschi A, Priano L, Mauro A, Riva G, Repetto C, Regalia C, Molinari E, Notaro P, Paolucci S, Sandrini G, Simpson SG, Wiederhold B, Tamburin S (2016) Psychological Treatments and Psychotherapies in the Neurorehabilitation of Pain: Evidences and Recommendations from the Italian Consensus Conference on Pain in Neurorehabilitation. Front Psychol 7:115

38. Reiner K Tibi L, Lipsitz JD (2013) Do mindfulness- based intervention reduce pain intensity? A critical review of the literature. Pain Med 14:230-242

39. Tonelli AN, Buse DC, Lipton RB (2011) Chronic migraine: epidemiology and disease burden. Curr Pain Headache Rep 15:70-78 
40. Wells RE, Burch R, Paulsen RH, Wayne PM, Houle TT, Loder E (2014) Meditation for Migraines: A Pilot Randomized Controlled Trial. Headache 54:1484-1495

41. Bakhshani NM, Amirani A, Amirifard H, Shahrakipoor M (2015) The Effectiveness of Mindfulness-Based Stress Reduction on Perceived Pain Intensity and Quality of Life in Patients With Chronic Headache. Glob J Health Sci 8:142-151

42. Day MA, Thorn BE, Ward LC, Rubin N, Hickman SD, Scogin F, Kilgo GR (2014) Mindfulness-based cognitive therapy for treatment of headache pain: A pilot study. Clin J Pain 30:152-161

43. Day MA, Thorn BE, Rubin NJ (2014) Mindfulness-based cognitive therapy for the treatment of headache pain: A mixed-methods analysis comparing treatment responders and treatment non-responders. Complement Ther Med 22:278-285

44. Omidi A, Zargar F (2015) Effects of mindfulness-based stress reduction on perceived stress and psychological health in patients with tension headache. J Res Med Sci 20:1058-1063

45. Cathcart S, Galatis N, Immink M, Proeve M, Petkov J (2014) Brief mindfulness-based therapy for chronic tension-type headache: A randomized controlled pilot study. Behav Cogn Psychother 42:1-15

46. Andrasik F, Grazzi L, D'Amico D, Sansone E, Leonardi M, Raggi A, SalgadoGarcia F (2016) Mindfulness and headache: a "new" old treatment, with new findings. Cephalalgia 36:1192-1205

47. Wells RE, Smitherman TA, Seng EK, Houle TT, Loder EW (2014) Behavioral and mind/body interventions in headache: Unanswered questions and future research directions. Headache 54:1107-1113

48. Grazzi L, Andrasik F, D’Amico D, Usai S, Kass S, Bussone G (2004) Disability in Chronic Migraine Patients With Medication Overuse: Treatment Effects at 1Year Follow-up. Headache 44:678-683

49. D'Amico D, Tepper SJ (2008) Prophylaxis of migraine: general principles and patient acceptance. Neuropsychiatr Dis Treat 4:1155-1167

50. Ferrari A, Baraldi C, Sternieri E (2015) Medication overuse and chronic migraine: a critical review according to clinical pharmacology. Expert Opin Drug Metab Toxicol 11:1127-1144

51. Zheng H, Chen M, Huang D, Li J, Chen Q, Fang J (2015) Interventions for migraine prophylaxis: protocol of an umbrella systematic review and network meta-analysis. BMJ Open 5:e007594

52. Forde G, Duarte RA, Rosen N (2016) Managing Chronic Headache Disorders. Med Clin North Am 100:117-141

53. Kabat-Zinn J (2013) Full catastrophe living, third edition: using the wisdom of your body and mind to face stress, pain, and illness. Random House, New York

54. Segal Z, Williams JM, Teasdale J (2002) Mindfulness-based cognitive therapy for depression: a new approach to preventing relapse. Guilford Press, New York

55. Kabat Zinn J, Lipworth L, Burney R (1985) The clinical use of mindfulness meditation for the self-regulation of chronic pain. J Behav Med 8:163-190

56. Nash JM, McCrory DC, Nicholson RA, Andrasik F (2005) Efficacy and effectiveness approaches in behavioral treatment trials. Headache 45:507-512

57. Kosinski M, Bayliss MS, Bjorner JB, Ware JE Jr, Garber WH, Batenhorst A, Cady R, Dahlöf CG, Dowson A, Tepper S (2003) A six-item short-form survey for measuring headache impact: the HIT-6. Qual Life Res 12:963-974

58. Stewart WF, Lipton RB, Whyte J, Dowson A, Kolodner K, Liberman JN (1999) An international study to assess reliability of the Migraine Disability Assessment (MIDAS) score. Neurology 53:988-994

59. Andrasik F, Holroyd KA (1980) Reliability and concurrent validity of headache questionnaire data. Headache 20:44-46

60. Beck AT (1967) The diagnosis and management of depression. Universisty of Pennsylvania Press, Philadelphia

61. Furlanetto LM, Mendlowicz MV, Romildo Bueno J (2005) The validity of the Beck Depression Inventory-Short Form as a screening and diagnostic instrument for moderate and severe depression in medical inpatients. J Affect Disord 86:87-91

62. Spielberger CD (1989) State-Trait Anxiety Inventory: Bibliography, 2nd edn. Consulting Psychologists Press, Palo Alto

63. Predabissi L (1989) Santinello M. Inventario per I'ansia di stato e di tratto Nuova versione italiana dello S.T.A.I. - Forma Y, Organizzazioni Speciali, Firenze

64. Buse DC, Silberstein SD, Manack AN, Papapetropoulos S, Lipton RB (2013) Psychiatric comorbidities of episodic and chronic migraine. J Neurol 260: 1960-1969
65. Tfelt-Hansen P, Pascual J, Ramadan N, Dahlöf C, D'Amico D, Diener HC (2012) Guidelines for controlled trials of drugs in migraine: Third edition. A guide for investigators. Cephalalgia 32:6-38

66. Boudreau GP, Grosberg BM, McAllister PJ, Lipton RB, Buse DC (2015) Prophylactic onabotulinumtoxinA in patients with chronic migraine and comorbid depression: An open-label, multicenter, pilot study of efficacy, safety and effect on headache-related disability, depression, and anxiety. Int J Gen Med 9:79-86

67. D'Amico D, Grazzi L, Bussone G, Curone M, Di Fiore P, Usai S, Leonardi M, Giovannetti AM, Schiavolin S, Raggi A (2015) Are depressive symptomatology, self-efficacy, and perceived social support related to disability and quality of life in patients with chronic migraine associated to medication overuse? Data from a cross-sectional study. Headache 55: 636-645

68. Beck AT, Steer RA, Brown G (1996) BDI-II. Beck Depression Inventory: Second Edition. The Psychological Corporation, San Antonio

69. Meadows GN, Shawyer F, Enticott JC, Graham AL, Judd F, Martin PR, Piterman L, Segal Z (2014) Mindfulness-based cognitive therapy for recurrent depression: A translational research study with 2-year follow-up. Aust N Z J Psychiatry 48:743-755

70. Sundquist J, Lilja ̊, Palmér K, Memon AA, Wang X, Johansson LM, Sundquist $K$ (2015) Mindfulness group therapy in primary care patients with depression, anxiety and stress and adjustment disorders: randomised controlled trial. Br J Psychiatry 206:128-135

71. Mayor S (2015) Mindfulness based therapy is as effective as antidepressants in preventing depression relapse, study shows. BMJ 350:h2107

72. Chiesa A, Castagner V, Andrisano C, Serretti A, Mandelli L, Porcelli S, Giommi F (2015) Mindfulness-based cognitive therapy vs. psycho-education for patients with major depression who did not achieve remission following antidepressant treatment. Psychiatry Res 226(2-3):474-483

73. Kuyken W, Hayes R, Barrett B, Byng R, Dalgleish T, Kessler D, Lewis G, Watkins E, Brejcha C, Cardy J, Causley A, Cowderoy S, Evans A, Gradinger F, Kaur S, Lanham P, Morant N, Richards J, Shah P, Sutton H, Vicary R, Weaver A, Wilks J, Williams M, Taylor RS, Byford S (2015) Effectiveness and costeffectiveness of mindfulness-based cognitive therapy compared with maintenance antidepressant treatment in the prevention of depressive relapse or recurrence (PREVENT): a randomised controlled trial. Lancet 386: 63-73

74. Gaul C, Visscher CM, Bhola R, Sorbi MJ, Galli F, Rasmussen AV (2011) Team players against headache: multidisciplinary treatment of primary headaches and medication overuse headache. J Headache Pain 12:511-519

75. Kabat Zinn J (2003) Mindfulness-based interventions in context: past, present, and future. Clin Psychol Sci Pract 10:144-156

76. Pompili M, Di Cosimo D, Innamorati M, Lester D, Tatarelli R, Martelletti P (2009) Psychiatric comorbidity in patients with chronic daily headache and migraine: a selective overview including personality traits and suicide risk. $J$ Headache Pain 10:283-290

77. Pompili M, Serafini G, Di Cosimo D, Dominici G, Innamorati M, Lester D, Forte A, Girardi N, De Filippis S, Tatarelli R, Martelletti P (2010) Psychiatric comorbidity and suicide risk in patients with chronic migraine. Neuropsychiatr Dis Treat 6:81-91

78. Serafini G, Pompili M, Innamorati M, Gentile G, Borro M, Lamis DA, Lala N Negro A, Simmaco M, Girardi P, Martelletti P (2012) Gene variants with suicidal risk in a sample of subjects with chronic migraine and affective temperamental dysregulation. Eur Rev Med Pharmacol Sci 16:1389-1398

79. Breslau N (1992) Migraine, suicidal ideation, and suicide attempts. Neurology 42:392-395 